\section{Las Fintech y la inclusión financiera en la era digital: El impacto en la reducción de la pobreza y la informalidad en el Perú}

\author{
Fintech and financial inclusion in the digital era: The impact \\ on the reduction of poverty and informality in Peru
}

\begin{abstract}
RESUMEN
La inclusión financiera significa tener acceso a servicios que solucionen un problema o satisfagan una necesidad financiera de la población con la finalidad de reducir la informalidad y la pobreza. Estos servicios financieros son operaciones de transferencias de capitales, compra-venta de divisas, pagos de servicios, préstamos, ahorros, seguros, etc. De acuerdo con Scottish Executive (2005) Inclusión financiera es "El acceso para individuos a productos y servicios financieros apropiados. Esto incluye a las personas que tienen las habilidades, el conocimiento y la comprensión para hacer el mejor uso de esos productos y servicios. La exclusión financiera es a menudo un síntoma de pobreza, así como una causa". Los accesos a los servicios financieros contribuyen a crear oportunidades de negocio para la población generando ingresos sostenibles en el largo plazo con el objetivo de un mejor nivel de vida. La tecnología financiera es una herramienta importante para lograr estos fines, ya que permite fácil acceso a realizar transacciones a bajo costo y según las necesidades de los usuarios. En línea con lo mencionado, las startup fintech han cobrado gran importancia en los últimos años, ya que ellas han tomado como giro de negocio alguna de las principales actividades que realiza una institución financiera ofreciendo servicios en forma rápida, altamente especializada y a bajo costo. En el Perú (2017), existen altos niveles de pobreza 22\% e informalidad del PBI $18 \%$ y según estudios realizados por organismos multilaterales para disminuir estos indicadores podemos valernos de la tecnología; Sin embargo, en el campo del desarrollo tecnológico ocupamos los últimos lugares en el mundo, según el ranking de Institute for Management Development 2018. Palabras claves: Fintech; startup; inclusión financiera; tecnología digital; informalidad; pobreza.
\end{abstract}

\begin{abstract}
Financial inclusion means having access to services that solve a problem or satisfy a financial need of the population in order to reduce informality and poverty. These financial services are operations of capital transfers, purchase-sale of currencies, payments of services, loans, savings, insurance, etc. According to Scottish Executive (2005) Financial Inclusion is "Access for individuals to appropriate financial products and services. This includes
\end{abstract}

\section{Allan Vargas García}

avargasg@unmsm.edu.pe

Universidad Nacional Mayor de San Marcos, Facultad de Ciencias Administrativas. Lima, Perú 
people who have the skills, knowledge and understanding to make the best use of those products and services. Financial exclusion is often a symptom of poverty, as well as a cause". The access to financial services contribute to create business opportunities for the population, generating sustainable income in the long term with the objective of a better standard of living. Financial technology is an important tool to achieve these ends, since it allows easy access to perform transactions at low cost and according to the needs of users. In line with the above, fintech startups have gained great importance in recent years, since they have taken as a business line some of the main activities carried out by a financial institution offering services quickly, highly specialized and at low cost. In Peru (2017), there are high levels of poverty $22 \%$ and informality of GDP $18 \%$ and according to studies carried out by multilateral organizations to decrease these indicators we can use technology; however, in the field of technological development we occupy the last places in the world, according to the ranking of Institute for Management Development 2018.

Keywords: Fintech; startup; financial inclusion; digital technology; informality; poverty.

\section{INTRODUCCIÓN}

Según Bill \& Melinda Gates Foundation, "si resolvemos estos grandes problemas de inclusión financiera, será con nuevos modelos de negocios, tecnologías e innovaciones. Los datos nos permiten saber qué innovaciones funcionan y cuáles no" (citado en Gabor \& Brooks, 2017, p.1).

En los últimos años estamos presenciando el nacimiento de una nueva revolución industrial de nuevos emprendimientos tecnológicos que actúan junto con las instituciones tradicionales del sector financiero como los bancos, cajas municipales, empresas de seguros y otras redes de pagos.

Con los actores tradicionales de la industria, estos emprendimientos logran innovar introduciendo nuevos negocios disruptivos a través de los cuales ofrecen productos y servicios de manera más eficiente aprovechando las nuevas tecnologías tanto por su funcionalidad como por su bajo costo. Pero los beneficios no se limitan únicamente a la eficiencia; también ofrecen posibilidades de atraer a nuevos clientes -individuales o institucionalesal sistema financiero formal (Finnovista, 2017).

La tecnología digital con acceso a teléfonos móviles se incrementa en forma sostenida año a año y las plataformas web app (versión de página web optimizada y adaptable a cualquier dis- positivo móvil) están democratizando el acceso a servicios financieros.

Según estudios realizados por el Banco Mundial en el 2018, en países que han tenido avances en la inclusión financiera, se han evidenciado los siguientes obstáculos:

- Difícil acceso de la población por la lejanía en zonas rurales.

- Falta de conocimiento de los diferentes productos financieros.

- Falta de productos financieros para las necesidades del público.

- Carencia de marcos legales de protección al usuario.

- Adaptar los reguladores a las nuevas tecnologías.

- La falta de documento de identidad dificulta el financiamiento.

El Banco Mundial (2018) afirma: "Alrededor de 2,500 millones de personas no utilizan servicios financieros formales y el $75 \%$ de los pobres no tiene cuenta bancaria. La inclusión financiera es clave para reducir la pobreza e impulsar la prosperidad".

\section{OBJETIVO DEL ENSAYO}

El presente artículo busca integrar las Fintech y la inclusión financiera en la era digital en el 
Perú y su potencial para reducir la pobreza y la informalidad. Específicamente, el artículo tiene tres objetivos:

1. Primero, revisar la situación actual de la informalidad y su participación en la economía y el empleo.

2. Segundo, analizar la pobreza por área de residencia y región en el contexto de la exclusión financiera.

3. Tercero, incluir a estos conocimientos el desarrollo de la tecnología como herramienta útil para la inclusión financiera que tiene por objetivo la formalización y reducción de la pobreza.

\section{ARGUMENTACIÓN}

\section{Informalidad en el Perú.}

Una definición bastante utilizada según Soto (1989) es:

El sector informal está constituido por el conjunto de empresas, trabajadores y actividades que operan fuera de los marcos legales y normativos que rigen la actividad económica. Por lo tanto, pertenecer al sector informal supone estar al margen de las cargas tributarias y normas legales, pero también implica no contar con la protección y los servicios que el estado puede ofrecer.

El Banco Central de Reserva del Perú (2008) define:

La informalidad es motivo de preocupación porque refleja una ineficiente asignación de recursos (sobre todo de mano de obra) y una ineficiente utilización de los servicios del estado, lo cual podría poner en riesgo las perspectivas de crecimiento del país. La evidencia comparativa sugiere que la informalidad en el Perú es producto de la combinación de malos servicios públicos y un marco normativo que agobia a las empresas formales. Esta combinación se vuelve particularmente peligrosa cuando, como en el caso peruano, la educación y desarrollo de capacidades es deficiente, cuando los métodos de producción son aún primarios, y cuando existen fuertes presiones demográficas.
En el Perú existen millones de personas dedicadas a diferentes actividades, por ejemplo: choferes, jardineros, obreros, servicio de seguridad, etc., esta población cuenta con empleos informales o sub-formales y perciben su remuneración en efectivo. Este segmento de población no contribuye al sistema de salud, pensiones del seguro social o fondos de pensiones privados. El sector informal genera producción y crea puestos de trabajo para la economía. "En el año 2017, el PBI nominal asciende a 687 mil 037 millones de soles, de los cuales el 18,6\% fue generado por el sector informal, lo que significó una reducción en su participación de -0,3 puntos porcentuales respecto al año 2007".

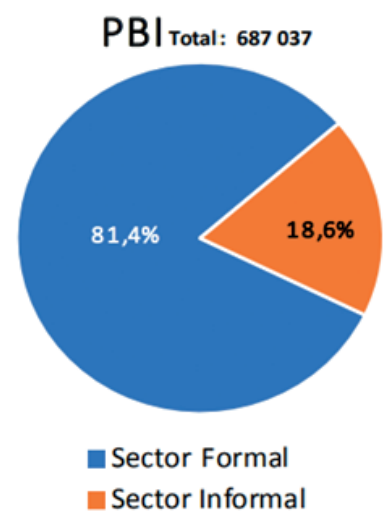

Figura 1. PERÚ: Participación del sector informal en el PBI, 2017 (Millones de soles).

Adaptado de "Producción y empleo informal en el Perú 20072017" por INEI, 2017.

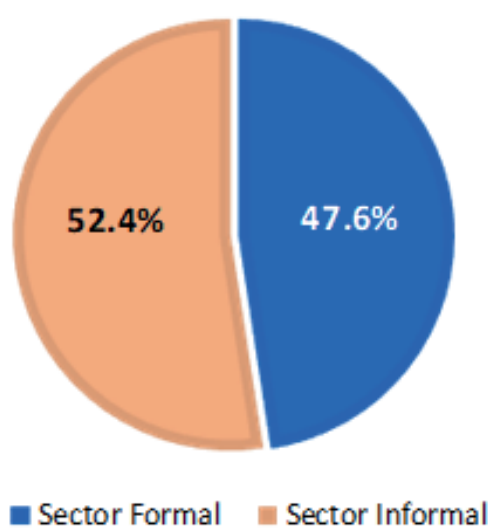

Figura 2. PERÚ: Participación del sector informal en el empleo, 2017 (Millones de soles).

Adaptado de "Producción y empleo informal en el Perú 20072017" por INEI, 2017.

La figura 3 muestra:

La evolución de la participación del sector informal en el PBI a precios corrientes y en el empleo equivalente, en el periodo 


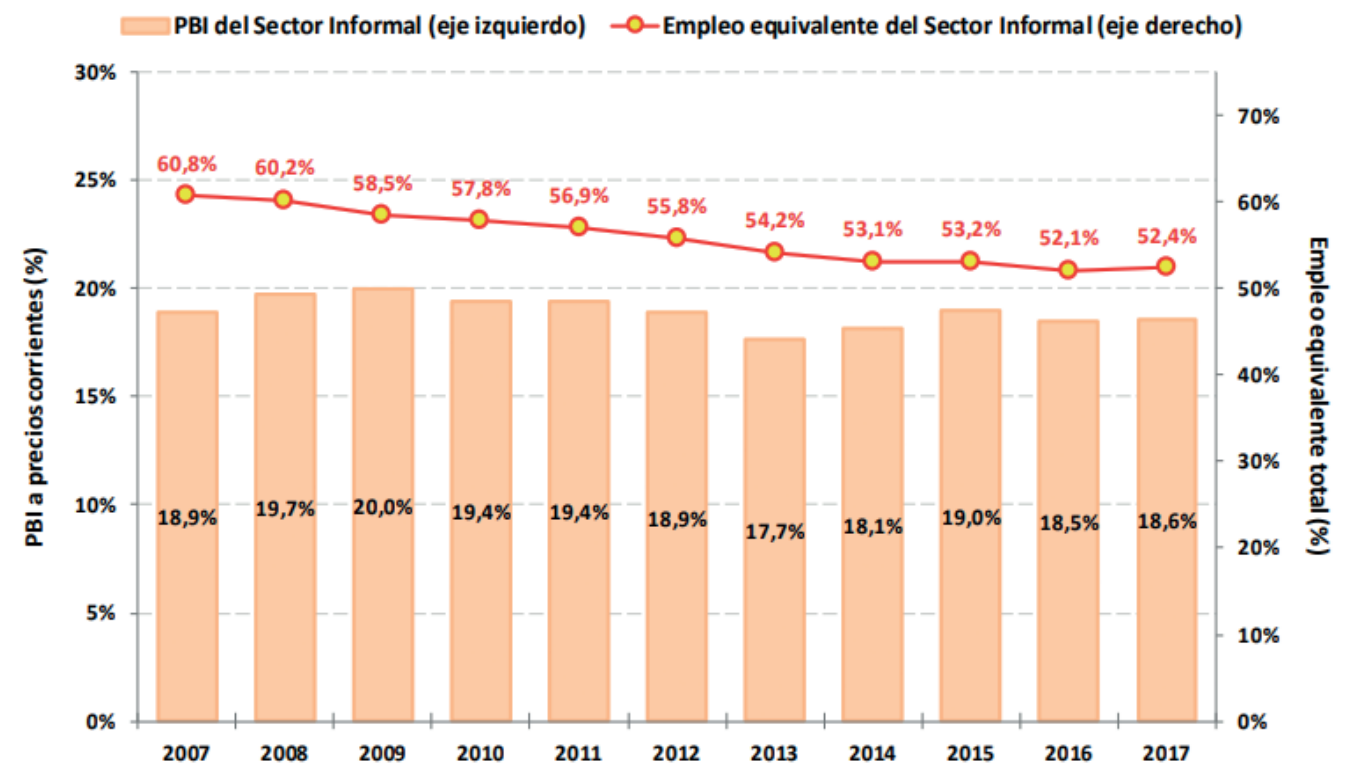

Figura 3. PERÚ: Participación del sector informal en el PBI y en el empleo, 2007-2017. Adaptado de "Producción y empleo informal en el Perú 2007-2017" por INEI, 2017.

2007-2017. En promedio, el sector informal aporta $18,8 \%$ al PBI, mientras que absorbe el 55,9\% del empleo total.

Entre 2007 y 2009 ocurrió un aumento de la participación del sector informal en el PBI, para luego descender, registrando su nivel más bajo en el 2013 con 17,7\%, produciéndose una reducción sostenida de su participación en el empleo equivalente. En el año 2017, la participación del sector informal en el PBI se incrementó levemente respecto al año anterior en $0,1 \%$. (Instituto Nacional de Estadística e Informática, 2018)

\section{Pobreza en el Perú}

"La pobreza es un fenómeno complejo que limita fuertemente la libertad de las personas y familias para desarrollar sus capacidades e integrarse a la sociedad, impidiéndoles realizarse y aportar sus propias riquezas a la comunidad donde viven." (Programa de las Naciones Unidas para el Desarrollo, PNUD 2015). "Pobreza es un fenómeno multidimensional que abarca aspectos materiales, no materiales, ingreso, salud, carencias relacionadas con el desarrollo humano como libertad, dignidad, autoestima, etc." (Comisión Económica para América Latina y el Caribe, 2003) La pobreza en el Perú se ha dividido en pobreza y pobreza extrema para lo cual se ha determinado parámetros para poder determinar la población que está dentro de este umbral. "La medida tradicional de pobreza compara los ingresos per cápita de los hogares con un punto de referencia o umbral, que se conoce como línea de pobreza. En términos generales, esta medición admite variantes absolutas y relativas" (Programa de las Naciones Unidas para el Desarrollo, 2015).

Para la determinación de la pobreza y pobreza extrema el INEI ha determinado que:

Para el año 2018, el valor de la línea de pobreza, es de S/. 338 per cápita mensual. Este valor constituye el valor mínimo mensual necesario que requiere una persona para satisfacer sus necesidades alimentarias y no alimentarias.

Además:

El valor de la línea de pobreza extrema es de S/.183 mensuales por cada persona que conforma un hogar, es decir, es el valor de los alimentos de una canasta socialmente aceptada, necesaria para cubrir un mínimo de requerimientos de energía. Entre los años 2017 y 2018, la línea de pobreza extrema no mostró variación alguna y se mantiene en S/.183. (INEI, 2018) 


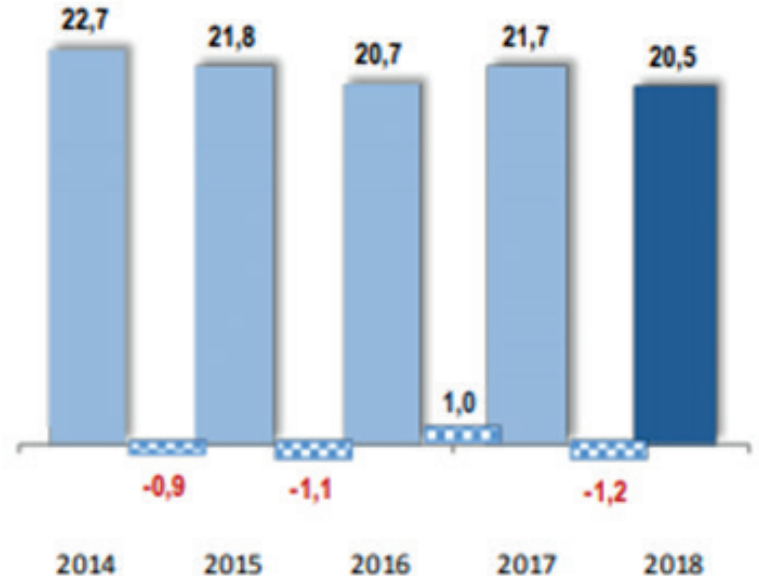

Figura 4. Evolución de la incidencia de la pobreza monetaria 20142018 (Porcentaje respecto del total de población)

Adaptado de "Evolución de la pobreza monetaria 2007-2018" por INEI, 2018.

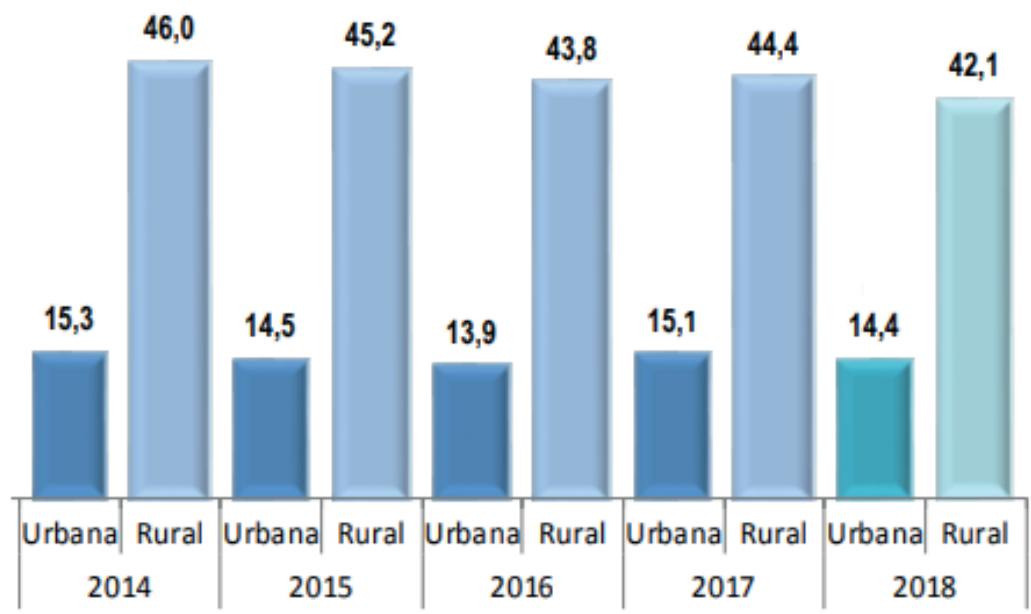

Figura 5. Evolución de la pobreza 2014-2018.

Adaptado de “Evolución de la pobreza monetaria 2007-2018” por INEI, 2018.

Según el INEI (2018):

El $20,5 \%$ de la población del país, que equivale en cifras absolutas a 6 millones 593 mil personas, se encontraban en situación de pobreza, es decir, tenían un nivel de gasto inferior al costo de la canasta básica de consumo compuesto por alimentos y no alimentos. Al comparar estos resultados con el nivel obtenido en el año 2017, se observa que la pobreza disminuyó en $1,2 \%$, que equivale a 313 mil personas pobres, menos que en el año 2017.

En la figura 5, también indicó, en el mismo año que:

Por área de residencia, la pobreza afectó más a los residentes del área rural, que incidió en el 42,1\% de su población, siendo tres veces más que en el área urbana $(14,4 \%)$. Al comparar con el año 2017, la pobreza disminuyó en 2,3\% en el área rural, en tanto, el área urbana lo hizo en 0,7\%. (INEI, 2018)

La figura 6 muestra:

A nivel de regiones naturales, la pobreza afectó al 30,4\% de la población de la Sierra, al $26,5 \%$ de la Selva, y al $13,5 \%$ de la Costa. Al comparar con el 2017 con el 2018, se observa que disminuyó en las tres regiones naturales del país, la Selva disminuyó en 2,1 puntos porcentuales, seguido de la Sierra en 1,2 puntos porcentuales y en la Costa en 0,9 punto porcentual. (INEI, 2018) 


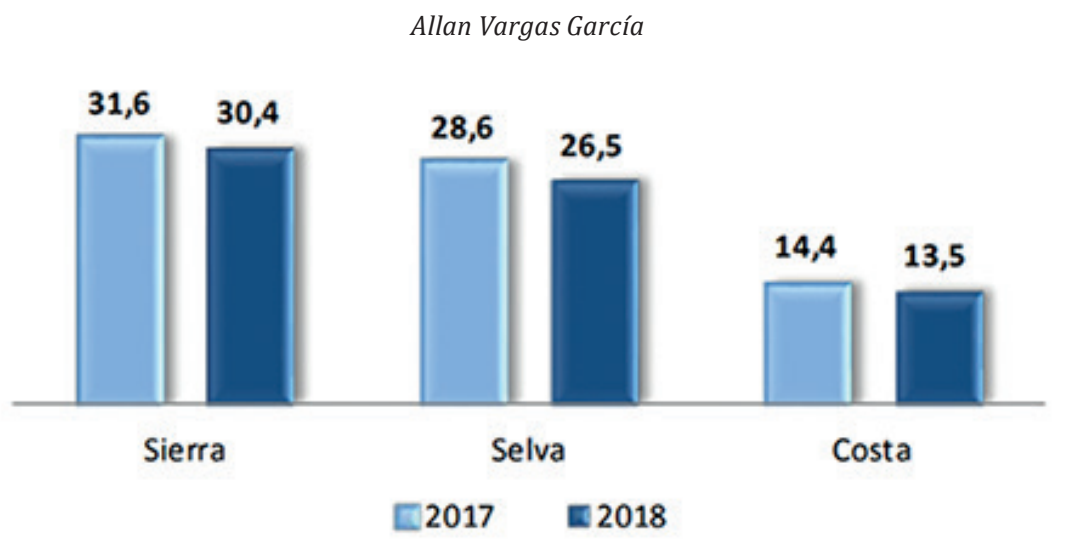

Figura 6. Incidencia de la pobreza monetaria total, Según región 2017-2018

(Porcentaje respecto del total de población de cada región).

Adaptado de “Evolución de la pobreza monetaria 2007-2018” por INEI, 2018.

Según el INEI (2017) "los departamentos con mayor incidencia de empleo informal son Huancavelica $(91,3 \%)$, Cajamarca $(90,1 \%)$ y Ayacucho $(88,9 \%)$, mientras que aquellos con menor incidencia de empleo informal es Lima, incluido Callao, $(58,5 \%)$ e Ica $(62,2 \%) "$.

Tabla 1

Perú: Incidencia del empleo informal por departamento, 2017

\begin{tabular}{lc}
\hline \multirow{2}{*}{ Departamento } & Empleo informal \\
\cline { 2 - 2 } Total & $72,5 \%$ \\
Huancavelica & $91.3 \%$ \\
Cajamarca & $90.1 \%$ \\
Ayacucho & $88.9 \%$ \\
Puno & $88.1 \%$ \\
Apurímac & $86.7 \%$ \\
San Martín & $85.6 \%$ \\
Huánuco & $85.4 \%$ \\
Amazonas & $84.9 \%$ \\
Cusco & $82.5 \%$ \\
Junín & $82.2 \%$ \\
Loreto & $80.7 \%$ \\
Áncash & $80.2 \%$ \\
Ucayali & $79.9 \%$ \\
Pasco & $79.2 \%$ \\
Piura & $77.7 \%$ \\
Tumbes & $77.3 \%$ \\
Madre de Dios & $76.2 \%$ \\
Lambayeque & $75.0 \%$ \\
Tacna & $73.2 \%$ \\
La Libertad & $72.4 \%$ \\
Moquegua & $68.8 \%$ \\
Arequipa & $65.4 \%$ \\
Lima 1/ & $62.2 \%$ \\
\hline & $58.5 \%$ \\
\hline
\end{tabular}

Nota: Porcentaje de empleo informal en el Perú por departamento. Adaptado de "Evolución de la pobreza monetaria 2007-2018" por INEI, 2018.

\section{La inclusión financiera en el Perú}

Según estudios del Banco Mundial (2017) los servicios financieros contribuyen a impulsar el desarrollo facilitando la inversión en educación, salud y negocios. Asimismo, tomar previsiones de formas de ahorro y manejar las emergencias financieras como quedarse sin ingresos o perdidas económicas, y de esa manera sea más difícil para las familias quedarse en la pobreza.

Según el índice Global Findex del Banco Mundial (2017) que mide la inclusión financiera en más de 140 países indica para el Perú, lo siguiente:

- El $42 \%$ de adultos tienen una cuenta en el sistema financiero.

- $\quad$ El 8\% tienen una cuenta de ahorros.

- $\quad$ El 28\% tienen una cuenta de débito.

- El 15\% tienen préstamos en instituciones financieras.

- El 12\% tienen tarjetas de crédito y

- El 15\% reciben sus sueldos con abono en cuenta.

Algunos problemas de la exclusión financiera:

- El trabajador no puede obtener una cuenta de pago de haberes.

- No se puede pagar los servicios públicos con cargo en cuenta.

- No puede acceder a préstamos de entidades financieras. 
Las Fintech y la inclusión financiera en la era digital: El impacto en la reducción de la pobreza y la informalidad en el Perú

- La población se obliga a aceptar financiamiento de informales.

- No se puede acceder a seguros formales personal o empresarial.

Respecto a la tecnología en la inclusión financiera, los datos para el Perú son:

- El 1\% reciben sus sueldos a través de web o móviles.

- El 5\% utilizan smartphones o web para acceder a una cuenta.

- El 3\% tienen cuenta de ahorros generado por móvil o web.

Los peruanos no tienen cuentas bancarias por las siguientes razones:

- El 20\% porque las instituciones financieras son muy lejanas.

- El 38\% porque los servicios financieros son costosos.

- El 19\% porque no reúnen la documentación necesaria.

- $\quad$ El 24\% porque no tienen confianza en instituciones financieras.

\section{La tecnología en el Perú}

El Institute for Management Development (IMD) mide: "la capacidad de un país en adoptar y explorar las tecnologías digitales que permitan una transformación en las prácticas gubernamentales, en los modelos de negocios y en la sociedad en general". El Ranking de Com- petitividad Digital Mundial del IMD incorpora tres pilares: (a) Conocimiento, (b) Tecnología, y (c) Preparación para el Futuro. El ranking mundial de competitividad digital estudia 63 economías. Estados Unidos lidera el ranking 2018 seguido por Singapur, Suecia, Dinamarca y Suiza. El Perú ocupa el puesto 60 de un total de 63 países en estudio (Ver Tabla 3 y Figura 7).

Según el ranking de IMD (2018):

El Perú mejora dos posiciones, del 62 al 60 , principalmente debido a un mejor desempeño en el pilar conocimiento, que pasa del puesto 62 al 60 ; y a una posición más estable en tecnología, que permanece en el puesto 57. Sin embargo, cae en dos posiciones en el pilar preparación para el futuro, del puesto 58 al 60 .

Como podemos apreciar el Perú se encuentra en los últimos lugares del mundo respecto a competitividad digital (lugar 60 de 63 economías) mostrando fragilidad en los tres pilares de evaluación. Para revertir el rezago se debe realizar grandes cambios a nivel de gobierno como mejorar la red de conectividad a nivel nacional con la red de fibra óptica $(2,5 \mathrm{ghz})$, y fomentar la cultura digital en la población a través de campañas de educación en el uso de herramientas tecnológicas.

Joseph Stiglitz-Nobel de Economía (2018) señala: "Tenemos que entender mejor todas estas nuevas tecnologías para tener una visión clara hacia dónde vamos, la revolución digital es una oportunidad de reducir la desigualdad de las sociedades".

Tabla 2

Metodología de la encuesta Global Findex 2017

\begin{tabular}{|c|c|c|c|c|c|c|}
\hline País & Región & Periodo de recolección de datos & Entrevistas & Margen de error & Modo de entrevistas & Lenguaje \\
\hline Perú & Latam & 1-25 Jul, 2017 & 1,000 & 3.8 & Cara a cara & Español \\
\hline \multicolumn{7}{|c|}{ Nota: Encuesta Perú. Adaptado de “Globalfindex Database” por Banco Mundial, 2017.} \\
\hline \multicolumn{7}{|l|}{ Tabla 3} \\
\hline \multicolumn{7}{|c|}{ Perú: Ranking general y factores de competitividad digital mundial 2018} \\
\hline \multicolumn{2}{|c|}{ Overall \& Factors } & 2014 & 2015 & 2016 & 2017 & 2018 \\
\hline \multicolumn{2}{|c|}{ Overall } & 59 & 57 & 58 & 62 & 60 \\
\hline \multicolumn{2}{|c|}{ Knowledge } & 58 & 58 & 61 & 62 & 60 \\
\hline \multicolumn{2}{|c|}{ Technology } & 54 & 52 & 53 & 57 & 57 \\
\hline \multicolumn{2}{|c|}{ Future readiness } & 55 & 56 & 55 & 58 & 60 \\
\hline
\end{tabular}

Nota: Competitividad Perú. Adaptado de “World Competitiveness Center”, por Institute for Management Development, 2018. 


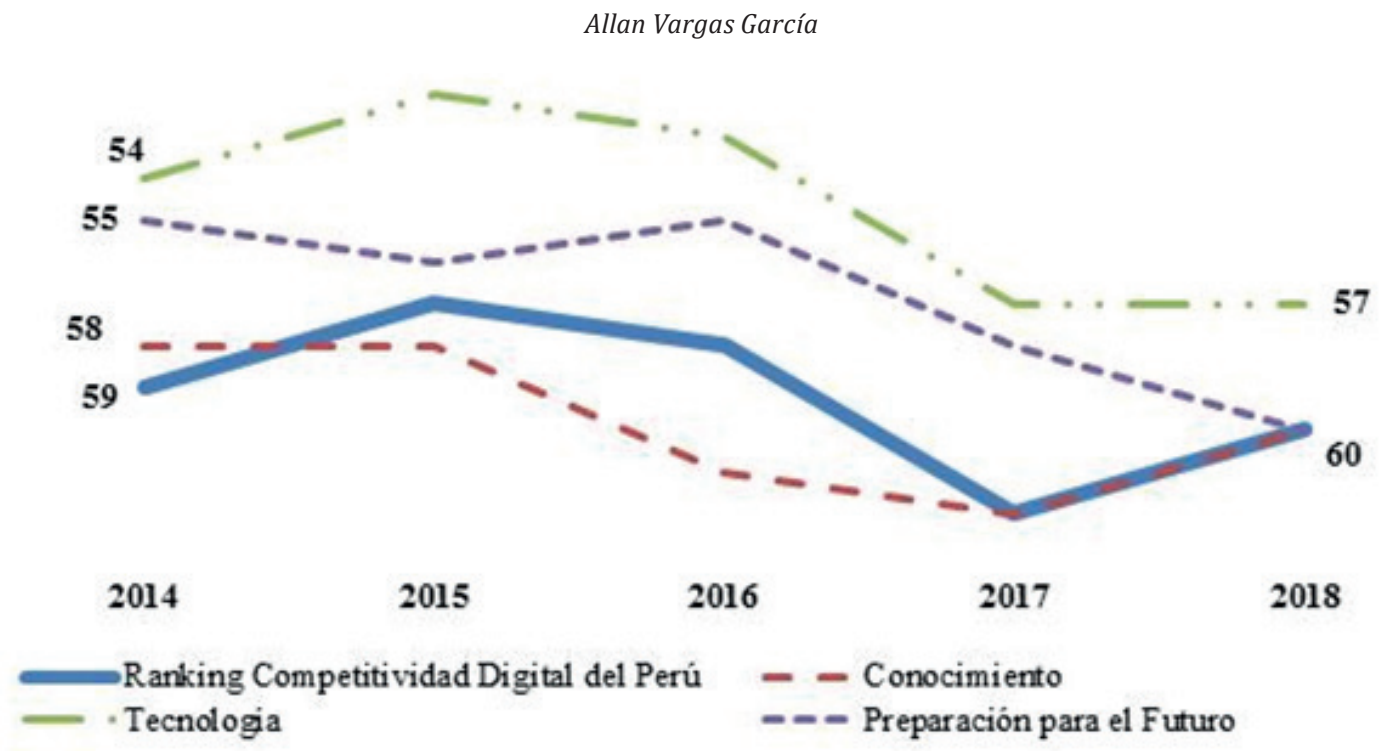

Figura 7. Resultado general y por factor de Perú 2014-2018.

Adaptado de "World Competitiveness Center", por Institute for Management Development, 2018.

\section{Las Fintech (tecnología financiera)}

La tecnología está cobrando gran importancia en todas las actividades que realiza el ser humano, y así también en el caso de las finanzas. Las empresas Fintech son empresas tipo startups de alto nivel tecnológico que se han especializado en una de las actividades que realizan las instituciones financieras de tal forma que son de reacción rápida, de bajo costo y apuestan por la captación de nuevos clientes. Estas empresas están revolucionando el sector financiero con un nuevo modelo de negocio y de propuesta de valor para otorgar soluciones a problemas o satisfacción de necesidades.

Francisco Gonzales, Chief Executive Officer del Banco BBVA (2018) señala: "Con la revolución digital habrá más riqueza y bienestar, y menos desigualdad, después de cada revolución industrial, siempre se ha creado más riqueza y más bienestar para toda la humanidad". Según el Banco Interamericano de Desarrollo BID (2018) en su publicación Fintech América Latina 2018, se identifican 1,166 emprendimientos Fintech a lo largo de 18 países de América Latina, aunque 5 países concentran el $86 \%$ del total de la actividad Fintech de la región:

- Brasil, con 380 Fintech (33\% del total).

- México, con 273 Fintech (23\% del total).

- Colombia, con 148 Fintech (13\% del total).

- Argentina, con 116 Fintech (10\% del total).

- Chile, con 84 Fintech (7\% del total).
En los últimos puestos están 4 países que acumulan entre el 1\% y el 5\% del total:

- Perú con 57 Fintech (5\% del total).

- Ecuador con 34 Fintech (3\% del total).

- Uruguay con 28 Fintech (2\% del total).

- Venezuela con 11 Fintech (1\% del total).

Por otro lado, las instituciones del sector público encargadas de realizar la función de regular las actividades de los emprendimientos Fintech se ven sobrepasadas por un número cada vez mayor de empresas financieras digitalizadas y se ven imposibilitadas de cumplir con sus funciones de supervisión. Se requiere una transformación del aparato estatal de regulación que automatice sus procesos para cumplir con su función de mitigar los riesgos del público usuarios de los servicios financieros y transparentar las actividades de la Fintech.

Para alcanzar mayores avances en la inclusión financiera es necesario crear un entorno normativo con reglamentación que facilite el desarrollo de nuevos negocios digitales tanto de instituciones financieras tradicionales como no tradicionales; asimismo, la normatividad debe estar dirigido a la protección de los usuarios y a la educación financiera.

\section{CONCLUSIONES}

- La tecnología permite incentivar la inclusión financiera y lograr que los peruanos 
participen del sistema financiero. Los indicadores de inclusión financiera en el Perú $42 \%$ se encuentran por debajo de los indicadores de los países de LATAM y de las principales economías a nivel mundial. Para lograrlo, se deberá respaldar uso de la internet en las plataformas web y móvil principalmente en zonas rurales del país.

- Difundir en la población el uso de dinero electrónico, a través de la billetera electrónica, efectivo móvil, transferencias propias y de terceros, entre otros. Según el Banco Central de Reserva en el Perú los pagos con efectivo representan el $90 \%$ de los pagos minoristas (2017). Las desventajas de la dependencia del manejo del dinero en efectivo están relacionadas con la inseguridad por robos, uso inapropiado, falsificaciones, fraudes, lavado de activos, etc.

- Identificar los obstáculos que impiden la expansión de los servicios financieros en el país como: las condiciones geográficas, la excesiva cantidad de documentos solicitados por las instituciones, los altos costos de los servicios financieros y la falta de confianza de los usuarios; estos factores asociados a la falta de información crediticia y los marcos legales estáticos complican el panorama de inclusión financiera en el Perú.

- La limitada inclusión financiera en el Perú está ligada al nivel de competitividad digital, ya que ocupa el puesto 62 en el mundo. Mientras no se mejore este indicador el efectivo seguirá siendo la principal fuente de pagos de las transacciones en la economía nacional.

\section{REFERENCIAS BIBLIOGRÁFICAS}

Banco Central de Reserva del Perú - Revista Estudios Económicos (2008). Causas y consecuencias de la informalidad en el Perú. Recuperado de http://www.bcrp.gob.pe/docs/Publicaciones/Revista-Estudios-Economicos/15/Estudios-Economicos-15-3.pdf

Banco Mundial (2018). Inclusión financiera. Recuperado de https://www.bancomundial.org/es/ topic/financialinclusion/overview
Banco Mundial (2018). Poverty and Equity Data. Recuperado de http://povertydata.worldbank. org/poverty/home/

BBVA (2018). Revolución digital. Recuperado de https://www.bbva.com/es/con-la-revolucion-digital-habra-mas-riqueza-y-bienestar-y-menos-desigualdad/

BBVA (2018). Stiglitz reflexiona sobre los retos sociales de la revolución digital. Recuperado de https://www.bbva.com/es/el-nobel-stiglitz-reflexiona-sobre-los-retos-sociales-de-la-revolucion-digital/

Comisión Económica para América Latina y el Caribe-CEPAL. (2003). Calidad de Vida de Adultos Mayores: Instrumentos para el Seguimiento Políticas y Programas. Recuperado de https://www.cepal.org/celade/noticias/paginas/7/13237/PP17.pdf

Finnovista-BID (2018). FINTECH: América Latina 2018 Crecimiento y consolidación. Recuperado de https://www.finnovista.com/informe-fintech-2018/

Gabor, D. y Brooks, S. (2017), "The digital revolution in financial inclusion: international development in the fintech era", New Political Economy. doi:10.1080/13563467.2017.1259298

IMD (2018). World Competitiveness Center Ranking. Recuperado de https://www.imd.org/ wcc/world-competitiveness-center-rankings/world-digital-competitiveness-rankings-2018/

Instituto Nacional de Estadística INEI (2018). Evolución de la pobreza monetaria 2007-2017 - Informe Técnico. Recuperado de https://www.inei. gob.pe/media/cifras_de_pobreza/informe_tecnico_pobreza_monetaria_2007-2017.pdf

Instituto Nacional de Estadística INEI. (2017). Perú: Producción y Empleo Informal en el Perú. Recuperado de https://www.inei.gob.pe/media/ MenuRecursivo/publicaciones_digitales/Est/ Lib1589/libro.pdf

Programa de las Naciones Unidas para el Desarrollo-PNUD. (2015). Medición de la Pobreza. Recuperado de http://www.cl.undp.org/content/ dam/chile/docs/pobreza/undp_cl_pobreza_comision_pobreza_2014.pdf

Scottish Executive. (2005) 'Financial Inclusion Action Plan', Recuperado de http://www.scotland. gov.uk/Resource/Doc/35596/0024808.pdf 